\title{
Linx
}

Revue des linguistes de l'université Paris X Nanterre

12 | 2002

«Comme la lettre dit la vie»

\section{Huon de Bordeaux : le plaisir du texte}

François Suard

(2) OpenEdition

Journals

Édition électronique

URL : http://journals.openedition.org/linx/1315

DOI : 10.4000/linx.1315

ISSN : 2118-9692

\section{Éditeur}

Presses universitaires de Paris Nanterre

Édition imprimée

Date de publication : 1 octobre 2002

Pagination : 254-260

ISSN : 0246-8743

Référence électronique

François Suard, «Huon de Bordeaux : le plaisir du texte», Linx [En ligne], 12 | 2002, mis en ligne le 10 octobre 2012, consulté le 01 mai 2019. URL : http://journals.openedition.org/linx/1315 ; DOI :

$10.4000 / \operatorname{linx} .1315$

Ce document a été généré automatiquement le 1 mai 2019.

Département de Sciences du langage, Université Paris Ouest 


\title{
Huon de Bordeaux : le plaisir du texte
}

\author{
François Suard
}

1 Décidément, la chanson de geste de Huon de Bordeaux, publiée il y a bien longtemps dans la magnifique édition de Pierre Ruelle ${ }^{1}$, et dont William Kibler est en train de préparer une nouvelle édition dans la version du BNF. fr. 22555, n'a pas fini de nous étonner. Le travail remarquable de Marguerite Rossi ${ }^{2}$ a montré la richesse de ce texte, et le fait qu'il s'agit bien d'une chanson de geste, en dépit de tous les ingrédients nouveaux dont elle a été pourvue, parmi lesquels, au premier chef, les épisodes d'aventures et la présence du merveilleux lié au personnage d'Auberon. La traduction que je suis en train de préparer pour l'édition Kibler a de nouveau attiré mon attention sur la qualité humoristique de ce texte et sur la variété et la finesse des procédés rhétoriques et stylistiques mis en œuvre. Je voudrais en donner ici quelques exemples en les dédiant à Michèle Perret, que la rigueur du travail linguistique n'a jamais conduite à la sécheresse, et pour qui l'humour est vertu cardinale.

Le plaisant, dans Huon, ressortit le plus souvent au comique de situation, qui fait jouer les ressorts traditionnels de l'opposition, grâce à la succession de scènes contrastées ou d'attitudes opposées du personnage à l'intérieur de scènes données.

Ces contrastes sont le plus souvent articulés à la « foleté », c'est-à-dire à l'inconséquence du jeune homme, mais l'opposition peut être cultivée pour elle-même, avec la succession d'un ton élevé et d'un registre plus trivial, par exemple. Ainsi lorsque le héros, qui a réussi à pénétrer dans Dunostre, apprend que son adversaire, le géant Orgueilleux, est endormi, il se refuse, malgré les supplications de la jeune Sebille, à tuer le monstre dans son sommeil, de peur des reproches qu'on pourrait lui faire en haute cour. Puis il ajoute :

"Dieu, c'ai ge dit? Or sus je folz prouvez:

Nulz ne lou sceit fors Dieu de majesteit,

Maix devant Dieu ne doit nulz mal pancer. » (ms. 22555, 213c),

et la conclusion de ce débat intérieur au ton élevé est que le géant sera tout d'abord défié. Mais il l'est avec une violence que ne laissaient pas présager les scrupules précédemment énoncés, et cette violence est hautement réjouissante : aux insultes de Huon

"Fiex a putain, villiés u vous dormés? " (5004)

succède en effet une scène burlesque, où l'on voit le géant mettre tant de violence dans son réveil que le châlit est jeté à terre. 
4 Autre mise en œuvre d'un retournement de situation: la modification significative apportée à un motif classique, par exemple une description énumérative. L'arrivée de Huon à Babylone commence par l'évocation de la foule immense, occupée à des tâches diverses, que le héros rencontre en arrivant dans la ville. La description commence avec les deux derniers vers de la laisse 46 et se poursuit avec trois laisses parallèles très brèves :

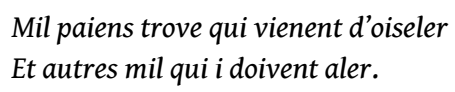

Dans ce passage, le poète se livre à un jeu subtil de répétitions et de variations. Quatre distiques, constituant un véritable refrain, reprennent le premier hémistiche de chaque vers, cet hémistiche étant différent dans le premier et le second vers du couplet : Mil en trova (v.1); Et autres mil (v.2). D'un vers à l'autre, le second hémistiche varie, mais cette variation est partielle et n'introduit pas véritablement d'autre activité. Il s'agit d'une modification de l'aspect temporel (la chasse au vol et le retour de la chasse, 1. 46; le ferrement des chevaus et ses préparatifs, le jeu et sa conclusion négative, 1. 47; le mouvement vers le palais ou la sortie de celui-ci, 1. 49).

Le groupe de trois vers de la 1.48 n'est lui-même qu'un distique bâti sur le modèle des quatre autres ; mais il est élargi par l'adjonction au premier vers d'une formule (sachiés a ensiant) qui permet de développer sur un vers entier (le v. 2) une activité qui ne prend ailleurs qu'un hémistiche ; le troisième vers reprend le schéma du v. 2 des distiques.

Il s'agit ici d'un jeu verbal qui, paraissant multiplier les informations, se limite en réalité à quelques aspects, dont plusieurs sont ludiques (la chasse, l'amour, le vin). C'est une ville joyeuse plutôt qu'inquiétante qu'on découvre, semblable à cette Orange « qui lors estoit mout belle » que Girart contemple dans la Chevalerie Vivien ${ }^{3}$ en revenant de Larchant :

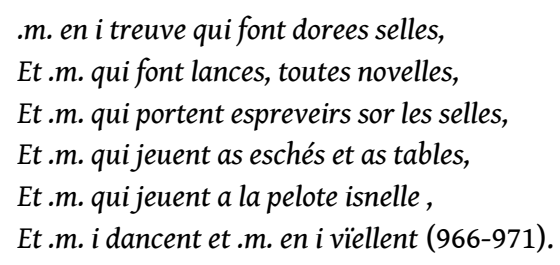


Mais de même qu'Orange la belle est aussi la ville qui va recevoir la terrible nouvelle de l'invasion sarrasine, notre texte se garde bien d'oublier la situation dramatique de son héros: Huon se trouve dans une ville à la puissance inquiétante, où sa vie est constamment en danger, et le poète termine effectivement là-dessus sa description en opérant, par un ultime jeu d'opposition, un changement de point de vue qui efface tout aspect plaisant et conclut sur le caractère écrasant de la foule ; le « regardant » devient le point de mire de tous ceux qu'il a regardés :

Tout chil millier esgarderent Huon (5444).

Ce regard collectif, anonyme, représente la tentative originale du poète pour faire sentir la masse qui entoure Huon. De plus, il annonce l'incident dramatique qui causera la captivité du héros : le regard des païens, renvoyant au héros le sien propre, lui fait oublier le talisman qu'il porte au bras, cet anneau gigantesque pris à l'Orgueilleux et qui pourrait lui servir de sauf-conduit pour entrer dans le palais de Gaudisse. Par «foleté » (5461), il ment au passage du premier pont, en prétendant qu'il est païen, et perd ainsi provisoirement - l'amitié d'Auberon.

Les exemples savoureux de retournements accompagnés de jeux verbaux sont souvent liés à la relation amoureuse, et concernent aussi bien les personnages féminins que Huon lui-même. Dès le départ, du reste, la mission de Huon est ambiguë, puisque parmi les obligations qui lui sont faites, on trouve celle des trois baisers qui doivent être donnés à la fille de l'émir. Péril et plaisir ne peuvent manquer d'être associés dans une telle activité, mais c'est d'abord chez la jeune fille que l'ambiguïté est manifeste. Esclarmonde s'évanouit - d'aise - lorsque Huon accomplit l'article de sa mission qui la concerne. La dame, tout d'abord, rassure son père, mais en proférant un mensonge, lorsqu'elle l'assure qu'elle guérira facilement du malaise qu'elle a éprouvé : « bien porai respaser » $(5722)$; un peu plus tard, elle se confie à la femme qui la sert :

"Sa douce alaine m'a si le cuer emblé

Se jou ne l'ai anuit a mon costé,

G'istrai dou sens ains qu'il soit ajorné» (5728-30)4.

11 S'il s'agit d'une maladie, ce n'est pas celle à laquelle pouvait penser Gaudisse.

12 Même dissimulation féminine chez la fille de l'émir Yvorin de Monbrant, joueuse d'échecs imbattable, sauf si l'amour sait la dompter. Or la beauté de Huon lui a tellement été vantée qu'elle n'a garde de trouver déshonorante la condition qui lui sera faite si jamais elle est battue par le bel arrivant :

"Et s'il vous puet, dame, du ju torner,

Entre ses bras toute une nuit girés

Et si fera de vous ses volentés. » (7498-500).

13 Avant même d'avoir aperçu Huon, elle est bien décidée à ce qu'un «si biaus hom » (7503) ne soit pas mis à mort à cause d'elle, ce qui ne l'empêche pas de feindre d'accepter les ordres de son père tout en se disant à elle-même son véritable désir :

"Vauroie je ja ke li gus fust finé,

Si me tenist dejouste son costé

Et puis fesist toute sa volenté. » (7519-21).

14 En fait, et c'est une autre subtilité du traitement du suspens, la dame n'aura même pas à choisir d'être vaincue : elle le sera par elle-même, ou plutôt par la beauté de Huon. Le poète fait en effet se succéder deux phases dans le jeu; dans la première, comme on peut s'y attendre, le héros est en mauvaise posture devant un adversaire émérite, et la dame se 
moque de lui : qu'il prenne bien garde, car sa vie est en grand péril. Mais Huon riposte avec hauteur : c'est la dame qui est près du déshonneur

"Quant en mes bras toute nue gerrés,

Qui sui sergans d'un povre menestrel » (7552-53).

Or ce déshonneur, nous le savons, n'en est pas un pour la dame, mais bien l'objet de son attente : cette menace délicieuse, jointe au pouvoir qui est celui de la vision du jeune homme, prive la joueuse de son savoir, la fait «mesgarder» (7558) et mater. S'en plaindra-t-elle? Non pas elle, mais son père, qui n'aurait jamais pensé que sa fille puisse être battue. Quant à la dame, elle attend sans doute son châtiment si plaisant, mais le cruel Huon, jouant les défenseurs de l'honneur de l'émir et de sa fille, repousse bien loin une telle solution: coup de théâtre pour la belle, qui s'en va "a cuer iré » (7577) et regrette son fol désir ; si elle avait su la conclusion de l'affaire, à coup sûr elle aurait usé de tout son savoir et battu un si pleutre adversaire. ${ }^{5}$

Autres types d'oppositions, celles qui s'organisent autour des relations entre Huon et son protecteur, le nain Auberon. Certaines sont de nature héroïque, telles les interdictions posées par le roi fée de tenter une épreuve périlleuse, comme le séjour à Tormont (3897-3917) ou l'entrée dans Dunostre (4578-4616). A chaque fois, Huon passe outre ; pour Tormont, on n'aboutit pas à une crise, puisqu'Auberon accepte de porter secours au héros si celui-ci en a besoin, pourvu qu'il ne l'appelle pas en vain. Pour Dunostre au contraire, l'opposition subsiste, car Auberon s'engage vigoureusement à ne pas intervenir, même si le cor l'appelle :

"Tu pués asés et tentir et sonner,

Mais par moi n'eres secourus ne tensés. " (4634-35).

Ces oppositions, et surtout la seconde, n'ont évidemment pour but que de mettre en valeur l'extraordinaire audace de Huon - audace toujours couronnée de succès - dans sa quête d'aventure héroïque, avec comme résultat le succès là où Auberon lui-même a échoué :

«Il me toli et la tour et l'ostel,

Et aveuc çou un bon hauberc safré » (4601-2).

L'opposition révèle ici une rivalité profonde mais féconde : pour que le héros s'affirme, il convient que le maitre s'efface, au moins provisoirement.

De telles oppositions sont moins plaisantes que signifiantes; d'autres au contraire nous ramènent au registre comique : il s'agit notamment de ce qui tourne autour de l'interdit solennel du manquement à la vérité et à la loyauté, ou à l'interdit sexuel proféré avant le départ de Babylone.

Préoccupé par la foule immense qu'il a contemplée dans la ville, Huon a menti au portier qui l'interrogeait au franchissement du premier pont: il s'est présenté comme un Sarrasin, afin de pouvoir passer, alors qu'il n'avait nullement besoin de le faire, puisqu'il possède un talisman qui peut lui ouvrir toutes les portes, l'anneau du géant Orgueilleux. Il se rend compte aussitôt qu'il a commis une faute, et qu'Auberon l'en punira. Dans un premier temps, il espère que le petit roi n'en saura rien (5509); puis il se décide à tenter l'épreuve du cor, qui lui montrera si Auberon est prêt à venir à son secours. La preuve est bientôt faite : le nain merveilleux ne répond pas.

21 Huon décide alors d'accomplir sa mission coûte que coûte, en se confiant à la Vierge. L'anneau de l'Orgueilleux le protège longtemps, mais la franchise retrouvée du jeune 
homme - il avoue avoir tué l'Orgueilleux (5792) - le fait capturer par les païens qui le jettent en prison.

C'est pour Esclarmonde le moment d'intervenir. Elle déclare son amour au jeune homme et lui demande d'accomplir sa volonté. Protestation vertueuse - et quelque peu présomptueuse - de Huon, qui ne peut que repousser les aimables perspectives qui s'offrent, puisque la dame est Sarrasine :

"Se devoie estre tos jors emprisonés

En ceste cartre, tant com porai durer,

Ne quier jou ja a vo car adeser » (5894-96).

Outrée, Esclarmonde décide de laisser le récalcitrant réfléchir, ou plutôt jeûner. Au bout de trois jours, Huon n'est plus aussi déterminé ; il maudit Auberon, implore le secours divin, et quand la dame entend ses lamentations, elle rabaisse un peu le niveau de ses demandes :

«Vous m'emmerriés o vous en vo regné,

Par Mahommet, je ne vous queroie el » (5926-27),

ce qui fait échapper l'acceptation de Huon au risque de péché mortel. Mais la déclaration plaisante qu'il fait alors va bien au-delà de la demande de la dame - qui n'a pas encore dit qu'elle veut se faire baptiser - et s'inscrit dans une opposition provocante et complète avec les affirmations premières du jeune homme :

"Se jou devoie tos les jors Diu flamer

Dedens infer, ens la cartre cruel,

Si ferai jou toute vo volenté » (5931-33).

La façon dont Huon répond plus tard à l'interrogatoire d'Estrument, le ménestrel qui le recueille après son naufrage, est moins sacrilège, car il ne s'agit plus alors de braver la discipline chrétienne, mais seulement et à nouveau les ordres d'Auberon; elle est pourtant tout aussi provocante par rapport à son objet - la sincérité - et s'inscrit dans un savoureux comique de situation. Sans ressource et furieux contre Auberon qui l'a, selon lui, injustement puni pour son union prématurée avec Esclarmonde, Huon est prêt à mentir autant qu'il le faudra :

«Puis qu'il m'i laise en si grant povreté,

Se mestiers est, je mentirai asés » (7170-71).

L'occasion se présente bientôt, car Estrument - dont Huon ignore tout, si ce n'est qu'il vient de lui permettre de se vêtir et de manger - lui demande quelle est son origine. Impossible de dire la vérité sur cette terre inconnue et probablement païenne; par nécessité et pour se venger d'Auberon, qui en a si mal usé avec lui, Huon choisit le mensonge :

«En vo despit mentirai jou asés;

Se m'as fait mal, je te ferai vieuté » (7241-42).

Et après menti une première fois, en attribuant son long silence, dû à une intense réflexion, à l'inattention

"Sire, fait il, que m'avés demandé?

Si m'aït Diex, je m'estoie oubliés » (7244-45),

il se fait passer pour un Africain, nommé Garinet ${ }^{6}$. Bien lui en prend, puisqu'Estrument, qui choisira plus tard par nécessité le camp chrétien, était au service de Gaudisse et maudit Huelin - qui est précisément en face de lui, mais qu'il ne peut connaître -, meurtrier de son mécène. 
Ce jeu sur le reniement des croyances ou des attachements n'est d'ailleurs pas le propre de Huon: l'émir Gaudisse, au moment où Huon se prépare à combattre Agrapart, le recommande à Mahomet, "son dieu »; mais comme l'on n'est jamais sûr de rien, il n'oublie pas le dieu de Huon :

«Et se li Dieus ke tu dois aourer

Vaut miex que chil que j'ai devant nommé,

Li plus vrais Dix te puist hui ramener. " (6543-45).

Reste enfin le parti amusant que le poète tire d'une formule répétitive, en la réintroduisant dans des contextes différents. C'est le cas pour l'évocation de la nudité de Huon, et aussi d'Esclarmonde, lorsque les jeunes gens, après la transgression de l'interdit sexuel, arrivent dans une île, chacun étant

Tout aussi nu comme au jor qu'il fu nés (6841).

Dans un tel contexte, la formule renvoie évidemment à la perte du statut remarquable des personnages, à une situation misérable qu'Esclarmonde désigne par le terme de vielté (6845). N'oublions pas qu'avant de s'unir à son amie en dépit des avertissements d'Auberon, Huon a passé en revue tous les avantages que Dieu lui a accordés («com m'avés viseté ! ", 6787) - , c'est-à-dire la possession des talismans du roi de féerie et l'amour d'Esclarmonde. Le fait de se retrouver dépouillé du moindre vêtement - «Si haut, si bas ", dira plus tard Huon, v. 7316 - dénonce après coup la forfanterie du héros et impose un contraste brutal. Ainsi privés de tout, les deux jeunes gens n'ont plus qu'à mourir, mais avec le réconfort que peut procurer l'amour et les grands exemples qu'il propose :

"Acolons nous, se morrons plus soef;

Tristrans morut por bele Iseut amer,

Si ferons nous, moi et vous, en non Dé. » (6848-50).

Faut-il toutefois prendre le passage entièrement au sérieux, et la compassion est-elle l'unique sentiment suggéré ? Il se pourrait bien qu'un regard humoristique, aussi, soit posé sur la situation de Huon, sinon sur celle d'Esclarmonde qui, elle, peut réellement faire figure de victime, puisqu'elle a protesté contre le dessein de son ami. Que l'union amoureuse suppose ou non la nudité - le texte évoque seulement l'acte de préparer un lit, puis l'étreinte, 6824-26 -, cette situation, serait-elle le fruit de la tempête qui a dispersé les (derniers) vêtements des amants, leur permet sans doute une proximité totale :

Sour une plance est Hues demorés,

Entre ses bras Esclarmonde al vis cler (6832-33),

mais elle ne peut manquer aussi d'être quelque peu importune au vu des circonstances. Contraste donc, et peut-être contraste plaisant.

Qu'en est-il maintenant des embrassements des jeunes gens, qui se sont réfugiés au creux des herbes et s'étreignent en invoquant le patronage des illustres amants ? Ici encore, il est vraisemblable qu'une certaine distance critique mine le sérieux qui pourrait accompagner la comparaison. Sans doute les proscrits de la forêt du Morrois ont-ils été en proie à la misère et à la solitude, sans doute leur nudité est-elle lisible dans le contreexemple que constitue la visite du roi Marc :

Sa chemise out Yseut vestue

(Se ele fust icel jor nue,

Merveilles lor fust meschoiet)

Et Tristan ses braies ravoit (1807-10) ${ }^{7}$ 
mais tout ceci se place au terme d'une longue histoire d'amour où les héros ont dû affronter la haine des barons jaloux. Or Huon n'a fait que céder ici à une pulsion juvénile, faite de désir immédiat et d'orgueil.

Même distance par rapport à l'autre épisode tristanien auquel on peut songer ici, celui de la mort des amants. D'abord, faut-il le rappeler, Tristan et Iseut sont alors habillés. D'autre part le contexte grandiose, qu'il s'agisse des causes de la blessure de Tristan, des circonstances de sa mort - le poids du destin ou de la jalousie -, des fortunes de mer qui retardent l'arrivée d'Iseut, enfin de la mort volontaire de la dame, est bien différent de celui qui entoure ces jeunes gens grelottants, mais qui ne songent qu'à vivre, comme le montre l'attitude de Huon qui, dès qu'il entend parler les pirates qui viennent de débarquer, se prépare à leur demander à manger.

On peut donc songer ici à la possibilité d'une lecture parodique, que la suite, avec la réitération de la formule-clé, ne contredira pas. C'est en effet « Tout aussi nus comme au jor que fu nés " (6868) que Huon, se présentant comme une victime de la tempête, se précipite vers les pirates qui viennent d'accoster, et obtient d'eux du pain. Nudité adjuvante ici, qui renforce la crédibilité de sa demande. Il n'en va pas longtemps ainsi, car les pirates, qui veulent en savoir davantage sur le jeune homme, le suivent et découvrent la dame " qui trestote nue ert » (6886) et la reconnaissent aussitôt pour la fille indigne de Gaudisse, qu'ils veulent conduire auprès de son oncle Yvorin afin de lui faire subir le châtiment de sa trahison.

La nudité de la dame apparaît ici comme un déguisement inefficace n'empêchant pas sa capture ; dans le même temps, la formule fait choc : serait-ce parce que la dame est nue qu'on peut aussitôt l'identifier? Elle n'a pourtant pas l'habitude, pour reprendre le titre d'un vaudeville connu, de se promener de la sorte.. Décidément, quelque chose manque à la dimension tragique.

Si la dame, grâce aux pirates qui la capturent, retrouve des vêtements, Huon, lui, est abandonné dans l'état où il se trouvait,

Tout aussi nu comme au jor que fu nés (6909),

avec toutefois une variation douloureuse, puisque les pirates, à défaut de le tuer, ont rendu sa situation plus précaire encore en lui bandant les yeux et en lui liant les poignets.

Quel espoir de salut peut avoir le jeune homme? Celui d'une intervention merveilleuse. Auberon ne peut la procurer lui-même, étant donné sa colère contre son protégé devenu rebelle, mais il peut faire connaître à ses propres amis la situation misérable du héros, ce qui suffira à attendrir Gloriant et surtout Malabron, un personnage protéiforme qui a déjà aidé Huon et l'aidera une fois encore. A nouveau, la nudité du héros est évoquée, mais elle participe cette fois clairement d'une épreuve douloureuse. Malabron pourra en effet arracher Huon à son île déserte, mais les ordres d'Auberon sont tels qu'il ne pourra rien lui donner ni lui ôter, ce qui signifie qu'il lui fera traverser la mer sans vêtements :

"Mais aussi nu con vous le troverés

Le porterés sor le rive de mer » (7081-82).

C'est donc «Tout aussi nuz com lou jour qu'il fuit nez» (7151) que le jeune homme s'installe sur la croupe du génie marin, et le ms. 22555, contrairement à celui de Tours, suivi dans l'édition Ruelle, insiste sur les souffrances endurées au cours de la traversée :

Ne vous sai dire conbien mist a passer,

La grant dollour Hue ait andurér ; 
En .xxx. lieu li est le sang vollez

Pour la froidure qu'il avoit en la mer (227c) ${ }^{8}$. montre avec quelle réticence il apporte son aide : il s'agit uniquement d'empêcher son ancien protégé de mourir. Et voici Huon errant de nouveau dans la nature, « Tout aussi nu comme au jor que fu nés » (7176). Cet état est à l'origine d'une nouvelle exploitation comique, lorsqu'Estrument le ménestrel, qui a disposé devant lui son repas, est épouvanté par la vision du jeune homme apparaissant devant lui ${ }^{9}$. L'infortuné est cette fois pris pour un monstre enfanté par les forces obscures de la nature, et Estrument de crier :

«Sauvages hom, ne me faites nul mel! " (7196).

La confusion est déjà plaisante en elle-même, car le ci-devant jeune chevalier, coutumier des exploits guerriers et initié depuis peu à l'amour, frigorifié de surcroît par sa traversée sur le dos de Malabron, n'a rien d'un familier des puissances chtoniennes. Mais l'erreur d'Estrument est soulignée de façon humoristique par le poète, qui fait jouer Huon avec la signification du terme sauvage :

«Par foi, dist Hues, sauvages sui asés;

Je ne vous quier touchier ne adeser,

Maix je vous prie de vous pain me donnez » (7197-7199) ; 作 homme et la façon dont elle s'est terminée. Devant Yvorin de Monbrant, Estrument relate les circonstances de sa rencontre avec Huon et n'omet pas la formule-refrain « Tout aussi nu comme au jor qu'il fu né » (7405). De façon plus dramatique, le ménestrel, sur le point d'être mis à mort, rappelle à Huon la bonté (8350) qu'il a eue

"Quant vous estiés en si grant povreté

Que trestous nus venis parmi le pré» (8352-53). Estrument, on s'en souvient, a aussi donné au jeune homme de quoi manger. Cette fois, la tonalité est grave, puisque le ménestrel est menacé de mort et que Garin de Saint-Omer va périr dans l'échauffourée. Elle met en avant la nécessaire gratitude de Huon dans une circonstance tragique, mais la formule itérative fait aussi ressurgir à l'arrière plan les circonstances héroï-comiques dans lesquelles elle a pu apparaître.

Dans Huon de Bordeaux, la maîtrise de "l'outillage épique", formule ou motifs, s'accompagne du plaisir avec lequel situations, personnages et discours sont manipulés. Le texte est bien celui d'une chanson de geste, mais la mise en œuvre est celle d'un parfait connaisseur de la littérature épique - la très longue allusion à la Chevalerie Ogier située au début du poème le montre bien, qui situe l'épopée entreprise en symétrie avec le texte cité $^{10}$.

e poète utilise le langage non seulement dans les perspectives habituelles, mais également comme un véritable jeu. Il s'agit toujours, bien sûr, de conter les gesta heroum, mais aussi de prendre, à certains moments, une distance avec ces exploits, et à jouer sur les situations et sur les mots. Cela signifie aussi que le texte - du moins celui de Huon n'est pas produit de hasard, résultat d'une inspiration liée éventuellement à la générosité du public (4986-91; 5512-19). C'est au contraire un patient travail littéraire exploitant 
notamment les effets comiques de la répétition et de l'opposition : en ce sens au moins, les appels à la générosité du public ${ }^{11}$ - que le ms. 22555 ne comporte pas - sont une plaisanterie de plus.

\section{NOTES}

1. Presses Universitaires de Bruxelles - Presses Universitaires de France, Bruxelles - Paris, 1960. Nos références, sauf indication contraire, renvoient à cette édition.

2. Huon de Bordeaux, Paris, Champion, 1975.

3. Voir l'éd. D. McMillan, Aix-en-Provence, CUER MA, 1997.

4. L'art de la dissimulation chez Esclarmonde se manifeste plus loin, mais d'une manière moins complexe, lorsqu'elle invoque un vœu fait à Mahomet pour ne pas coucher avec Galafre d'Aufalerne, qui l'a épousée (6956-64), ou lorsqu'elle attribue à une attaque de goutte l'émotion violente qu'elle éprouve en retrouvant Gériaume qui se présente à elle incognito (7961-67).

5. Elle renouvelle ses regrets plus tard, lorsque, depuis les murailles, elle contemple Huon qui se prépare à marcher contre Aufalerne : le jeune homme se serait montré courtois s'il l'avait au moins embrassée, et s'il avait demandé davantage, «Tous mes gens cors li fust abandonés, / S'en eüst fait toute sa volenté / Mais que mes peres l'eüst cent fois juré » (8049-51).

6. Le choix de ce nom, aussi peu « étranger » que possible, et digne de la poésie populaire ou de la farce, ajoute une note burlesque à la scène.

7. Voir Tristan et Iseut, édition de D. Lacroix et Philippe Walter, Paris, Lettres gothiques, 1989.

8. A comparer avec les vv. 7153-54 de l'édition Ruelle : «Et si a tant esploitié et erré / Que d'autre part a Huon arivé. »

9. La formule habituelle est à nouveau employée (7192).

10. Charlot, le fils de l'empereur, périt de la main du héros, alors que dans la Chevalerie c'est le fils du héros qui est tué par le même Charlot.

11. Vv. 4976-91, 5510-5519.

\section{AUTEUR}

FRANÇOIS SUARD

Université Paris 10 